\title{
A multi-wavelength study of the IRAS Deep Survey galaxy sample
}

\section{The far-IR properties}

\author{
P. Mazzei ${ }^{1}$, A. della Valle ${ }^{1,2}$, and D. Bettoni ${ }^{1}$ \\ 1 INAF, Padova Astronomical Observatory, Vicolo dell'Osservatorio 5, Padova 35128, Italy \\ e-mail: [paola.mazzei; antonio.dellavalle; daniela.bettoni]@oapd.inaf.it \\ 2 Department of Astronomy, Padova, Italy
}

Received 14 July 2006 / Accepted 13 September 2006

\section{ABSTRACT}

\begin{abstract}
Context. The luminosity function (LF) is a basic tool in the study of galaxy evolution since it constrains galaxy formation models. The earliest LF estimates in the IR and far-IR spectral ranges seem to suggest strong evolution. Deeper samples are needed to confirm these predictions. We have a useful IR data set, which provides a direct link between IRAS and ISO surveys, and the forthcoming deeper Spitzer Space Telescope and Akari cosmological surveys, to address this issue.

Aims. This data set allows us to derive the $60 \mu \mathrm{m}$ local LF to sensitivity levels 10 times deeper than before, to investigate evolutionary effects up to a redshift of 0.37 , and, using the $60 / 15 \mu \mathrm{m}$ bi-variate method, to analyze the poorly known $15 \mu \mathrm{m}$ local LF of galaxies. Methods. We exploited our ISOCAM observations of the IRAS Deep Survey (IDS) fields, to correct the $60 \mu \mathrm{m}$ fluxes for confusion effects and observational biases. We find indications of a significant incompleteness of the IDS sample, still one of the deepest farIR selected galaxy samples, below $\simeq 80 \mathrm{mJy}$. We have reliable identifications and spectroscopic redshifts for $100 \%$ of a complete subsample comprising 56 sources with $S(60 \mu \mathrm{m})>80 \mathrm{mJy}$.

Results. With our spectroscopic coverage we construct the $60 \mu \mathrm{m} \mathrm{LF}$ for a sample complete down to $80 \mathrm{mJy}$. This LF extends over three orders of magnitude in luminosity, from 9 up to more than 12 in $\log \left(L_{60} / L_{\odot}\right)$. Despite the fact that the redshift range of our sample exceeds $z=0.3$, the $V / V_{\max }$ test gives $\left\langle V / V_{\max }\right\rangle=0.51 \pm 0.06$, consistent with a uniform distribution of sources. A more direct test, whereby the LF was measured in each of four different redshift intervals, does not point out any signature of evolution. On the other hand, the rest-frame $15 \mu \mathrm{m}$ local LF we derive, extends up to $\log \left(L_{15} / L_{\odot}\right)=12$ and predicts 10 times more sources at $\log \left(L_{15} / L_{\odot}\right)=11$ than before.
\end{abstract}

Key words. galaxies: evolution - galaxies: ISM - galaxies: luminosity function, mass function

\section{Introduction}

Understanding how galaxies form and evolve is a key goal of modern physical cosmology. A fundamental observable of galaxies is their luminosity function (LF), which has long been used to constrain galaxy formation models and to quantify star formation and evolution both in luminosity and in density. The IR and far-IR spectral ranges are the best to deepen our knowledge of this subject since they trace the star formation that is responsible for galaxy formation. In particular, several satellite missions in the past (IRAS and ISO) and in the present (Spitzer, Akari) provided and will provide data that will be complementary for a detailed study of the LFs in such spectral domains.

The earliest IR estimate of the LFs, derived from the IRAS data (Rowan-Robinson et al. 1987; Saunders et al. 2000), indicated strong evolution, so that LF increases with redshift $\propto(1+z)^{3 \pm 1}$. Moreover, deep surveys at $15 \mu \mathrm{m}$ carried out with ISO (i.e., Elbaz et al. 1999; Flores 1999; Lari et al. 2001; Metcalfe et al. 2003) seem to require strong evolution of $15 \mu \mathrm{m}$ sources (Lagache et al. 2005, and references therein). Several evolutionary models were developed to explain these results (e.g., Franceschini et al. 2001; Rowan-Robinson 2001) and to fit the IR/submillimiter source counts with different degrees of success. Nevertheless, none of them is based on a local LF obtained from $15 \mu \mathrm{m}$ data, since the only available data until recently came from IRAS $12 \mu \mathrm{m}$ photometry (Rush et al. 1993;
Xu 1998; Fang et al. 1998). The first attempt to build up the $15 \mu \mathrm{m}$ LF of a NEPR subsample was made by Xu (2000). However, he said that it must be considered as a preliminary work because: i) the sample of galaxies used is an incomplete sample; ii) there is a possible misidentification between the sources in the $60 \mu \mathrm{m}$ redshift survey of Ashby et al. (1996) and the $15 \mu \mathrm{m}$ sources in his work (see della Valle et al. 2006, for more details); and iii) the model used to interpret the data treated all IR galaxies as a single population.

Our IDS/ISOCAM sample overcomes all these issues. It comprises a complete, $60 \mu \mathrm{m}$ selected sample of 56 galaxies in the North Ecliptic Polar Region (NEPR), a subsample of the original 98 IRAS Deep Survey (IDS) fields (Hacking \& Houck 1987). The IDS sample was defined by co-adding IRAS scans of the NEPR, representing more than $20 \mathrm{~h}$ of integration time (Hacking \& Houck 1987). It comprises 98 sources with $S(60 \mu \mathrm{m})>50 \mathrm{mJy}$ over an area of 6.25 square degrees.

Mazzei et al. (2001) exploited ISOCAM observations (range $12-18 \mu \mathrm{m}$ ) of 94 IDS fields (Aussel et al. 2000), centered on the nominal positions of IDS sources, to correct the $60 \mu \mathrm{m}$ fluxes for confusion effects and observational biases, finding indications of a significant incompleteness of the IDS sample below $\simeq 80 \mathrm{mJy}$. In della Valle et al. (2006), we presented spectroscopic and optical observations of candidate identifications of our ISOCAM sources. Combining such observations with those by Ashby et al. (1996), we have reliable identifications and 
spectroscopic redshifts for $100 \%$ of the complete subsample comprising 56 sources with $S(60 \mu \mathrm{m})>80 \mathrm{mJy}$. It is the deepest complete IRAS selected sample available and still one of the deepest complete far-IR selected samples. For comparison, the IRAS Point Source Catalog (hereafter PSC; Beichman et al. 1988) comprises about 250000 IR sources with a completeness limit of $0.5 \mathrm{Jy}$ at $60 \mu \mathrm{m}$ (Soifer et al. 1987). The deep ISOPHOT surveys, FIRBACK at $170 \mu \mathrm{m}$, (Puget et al. 1999; Dole et al. 2001), and ELAIS at $90 \mu \mathrm{m}$ (Oliver et al. 2000) are all complete down to about $100 \mathrm{mJy}$. Moreover, the $70 \mu \mathrm{m}$ Spitzer catalog of the $8.75 \mathrm{sq}$. deg. Bootes field is flux limited to $80 \mathrm{mJy}$ (Dole et al. 2004) and the Spitzer extragalactic "main" First Look Survey, covering about 4 sq. deg., is complete to about $20 \mathrm{mJy}$ at $70 \mu \mathrm{m}$ (cf. Fig. 2 of Frayer et al. 2006), but redshift measurements are available for a substantial fraction of sources (currently only $72 \%$ ) merely for $S_{70 \mu \mathrm{m}}>50 \mathrm{mJy}$.

Thanks to our ISOCAM and optical/near-IR observations, our sample, which provides a direct link between the IRAS and ISO surveys, and the forthcoming deeper Spitzer Space Telescope and Akari cosmological surveys ${ }^{1}$, is one of the farIR selected complete samples with the larger spectral coverage. In addition to the ISOCAM and to the $60 \mu \mathrm{m}$ fluxes, most of our sources $(\simeq 70 \%)$ have $100 \mu$ m fluxes, while the remaining $30 \%$ have upper limits, and several $(\simeq 40 \%)$ have $25 \mu \mathrm{m}$ fluxes from IRAS (Mazzei et al. 2001). Optical imaging has been already performed for $62.5 \%$ out of such a sample in at least one band, $B$ or $R$, and for $34 \%$ in both the bands (della Valle et al. 2006); moreover, Two Micron All Sky Survey (2MASS) data are available for $68 \%$ of the sample and VLA observations for a large fraction of these sources are also available (Hacking et al. 1989).

In this paper, which is the second step of our multiwavelength approach devoted to the study of the evolution of a far-IR selected sample of galaxies on which numerous studies of the far-IR evolution of galaxies still rely, we derive the $60 \mu \mathrm{m} \mathrm{LF}$ of such a sample. Our sample, ten times deeper in flux density than the PSC catalog, and thus less liable to be affected by the local density inhomogeneity, allows us to investigate evolutionary effects up to a redshift of 0.37 , five times deeper than the PSCz catalog $(z \leq 0.07$, Saunders et al. 2000). Moreover, we use the bi-variate method to translate the $60 \mu \mathrm{m}$ LF to the poorly known $15 \mu \mathrm{m}$ LF. We will compare our results with the recent determination of the $15 \mu \mathrm{m}$ local LF obtained by Pozzi et al. (2004) using the available data on the southern fields, S1 and S2, of the ELAIS survey (Oliver et al. 2000).

The paper is organized as follows: Sect. 2 focuses on farIR properties of our complete sample, Sect. 3 shows our derived $60 \mu \mathrm{m} \mathrm{LF}$, and Sect. 4 presents the $15 \mu \mathrm{m}$ LF based on the bi-variate $60 / 15 \mu \mathrm{m}$ method. In Sect. 5 there are our conclusions. Here and in the following we adopt: $\Lambda=0.7, \Omega_{\mathrm{b}}=0.3$, $H_{0}=70 \mathrm{~km} \mathrm{~s}^{-1} \mathrm{Mpc}^{-1}$.

\section{The far-IR properties}

Our complete sample comprises 56 IDS/ISOCAM sources. $25 \%$ out of these are beyond $z=0.1,12.5 \%$ beyond $z=0.15$, and

\footnotetext{
1 New deep observations of the NEPR are planned with the Akari space mission, also known as the InfraRed Imaging Surveyor (IRIS). It will map the entire sky in four far-IR bands, from 50 to $200 \mu \mathrm{m}$, and two mid-IR bands, at 9 and $20 \mu \mathrm{m}$, with far-IR angular resolutions of 25-45 arcsec, reaching a detection limit of $44 \mathrm{mJy}$ ( $5 \sigma$ sensitivity) in the 50-75 $\mu \mathrm{m}$ band (Pearson et al. 2004). With the Akari orbit, the integration time on the NEPR will be particularly high, and, correspondingly, the detection limit significantly deeper than average.
}
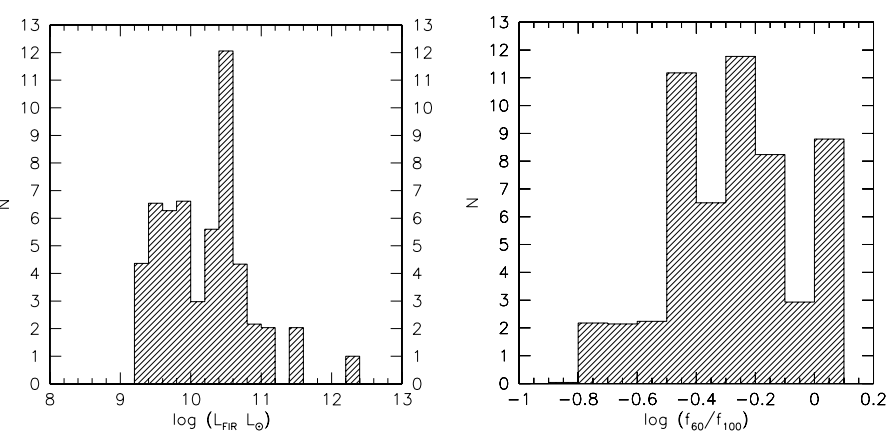

Fig. 1. Left: the rest-frame far-IR luminosity distribution of the IDS/ISOCAM sample of 56 galaxies. Here, as in Figs. 2-4, 7, 9, and 11, upper limits are taken into account by exploiting the Kaplan-Mayer estimator and by accounting for K-corrections and dust emission using evolutionary population synthesis models (see text). Right: the rest-frame distribution of the IRAS flux density ratio $f_{60} / f_{100}$, as revised in Mazzei et al. (2001), for the same sample.

only $5.3 \%$ at $z>0.2$ (della Valle et al. 2006). Such a sample is deeper than previous estimates (Ashby et al. 1996), showing a tail extending up to $z=0.375$, almost 4 Gyr in look-back time. Our morphological analysis (Bettoni et al. 2006) shows that, although $16 \%$ of our sources are multiple systems, unperturbed disk galaxies dominate the IDS/ISOCAM sample. One ULIRG, 3-53A, and two broad $\mathrm{H} \alpha$ emission line galaxies with AGN optical properties (i.e., 3-70A and 3-96A, see Bettoni et al. (2006) for more details) are also included in the complete subsample.

Figure 1 (left panel) shows the distribution of the far-IR luminosity ( $L_{\mathrm{FIR}}$, from 42.5 to $\left.122.5 \mu \mathrm{m}\right)$ of our sample, where $L_{\mathrm{FIR}}=4 \pi D_{L}^{2}(\mathrm{FIR}), \mathrm{FIR}=1.26 \times 10^{-14}\left(2.58 f_{60}+f_{100}\right) \mathrm{W} / \mathrm{m}^{2}$, and $f_{60}$ and $f_{100}$ are in Jy (Helou et al. 1988). In such a figure, as in the following ones, K-corrections were derived from evolutionary population synthesis models taking dust effects into account (Mazzei et al. 1995), luminosities were in units of solar bolometric luminosity, $L_{\odot}=3.83 \times 10^{33} \mathrm{erg} / \mathrm{s}$. Moreover, upper limits to flux densities were accounted for by exploiting the Kaplan-Meier estimator (Kaplan \& Meier 1958). Calculations were carried out using the ASURV v 1.2 package (Isobe \& Feigelson 1990), which implements methods presented in Feigelson \& Nelson (1985) and in Isobe et al. (1986). The Kaplan-Meier estimator is a non-parametric, maximumlikelihood-type estimator of the "true" distribution function (i.e., with all quantities properly measured, and no upper limits). The "survivor" function, giving the estimated proportion of objects with upper limits falling in each bin, does not produce, in general, integer numbers, but is normalized to the total number. This is why non-integer numbers of objects appear in the histograms of our figures.

The far-IR luminosity of the IDS/ISOCAM sample extends over 3 orders of magnitude (Fig. 1, left panel) with a mean value, $\log \left(L_{\mathrm{FIR}}\right)=10.2$, slightly lower than the mode of the distribution, $\log \left(L_{\text {FIR }}\right) \approx 10.5$. This value is almost the same as that of the Revised IRAS $60 \mu \mathrm{m}$ Bright Galaxy Sample (Sanders et al. 2003), and of a normal spiral galaxy, like the Milky Way (Mazzei et al. 1992, and references therein). The ULIRG galaxy, 3-53A, emits the maximum far-IR luminosity of the sample, nearly 100 times higher than the median value. In the same figure (Fig. 1, right panel) we present the rest-frame distribution of the $f_{60} / f_{100}$ flux density ratios for our sample. This ratio is a measure of the dust temperature, which gives information on the relative fraction of IR light from new and old star populations (Helou 1986; Mazzei et al. 1992). Its mean value, -0.3, 


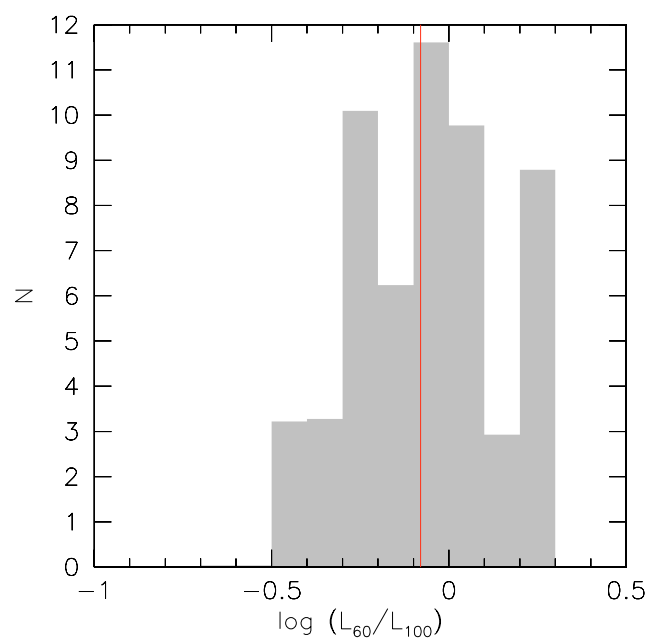

Fig. 2. The distribution of the rest-frame luminosity ratio, $\log L_{60} / L_{100}$, for our 56 IDS/ISOCAM sources; red continuous line corresponds to the value $\log L_{60} / L_{100}=-0.08$ (see text).

corresponds to a grain temperature of about $36 \mathrm{~K}$, consistent with the value observed for the bulk of IRAS galaxies (Sanders et al. 2003; Soifer et al. 1987). The $L_{60} / L_{100}$ ratio correlates with far-IR luminosity, as expected for the flux density ratio, $f_{60} / f_{100}$, but avoiding any redshift dependence, with the most luminous IRAS sources having the largest values of such a ratio (see Sanders \& Miraleb 1996, and references therein). From the distribution of the rest-frame luminosity ratio, $L_{60} / L_{100}$, we derive a mean value of -0.08 (Fig. 2). We divide the sample into two subsamples separated about this mean, i.e., a warm subsample of 24 IDS/ISOCAM sources with $\log \left(L_{60} / L_{100}\right) \geq-0.08$, and a cold subsample of 32 objects having $\log \left(L_{60} / L_{100}\right)<-0.08$ (Rowan-Robinson \& Crawford 1989). Figure 3 shows the far-IR luminosity distributions of warm and cold sources in our sample. Warm systems entail the overall luminosity range with a mode (mean) value, 10.45 (10.3), about 3 (1.5) times higher than that of cold sources.

We use the total far-IR luminosity to quantify the star formation rate (SFR). According to Chapman et al. (2000), $L_{\mathrm{TIR}}=k \times$ $S F R$ with $k$ ranging from 1.5 to 4.2 , in units of $10^{9} L_{\odot} M_{\odot} \mathrm{yr}^{-1}$, for Salpeter's IMF with upper and lower mass limits $100 M_{\odot}$ and $0.1 M_{\odot}$, respectively, and $L_{\mathrm{TIR}}=1.7 \times L_{60}$. We adopt $k=4.2$ by comparing the SFR as defined above with the Hopkins et al. (2003) calibration. The more luminous $60 \mu \mathrm{m}$ sources, i.e., those with higher SFRs, correspond to the more distant systems, 3-96 and 3-53, two warm sources (Fig. 4).

The dust temperatures suggest the presence of two galaxy populations: a spiral population with normal dust temperature (i.e., <36 K), mean $\mathrm{SFR} \simeq 6 M_{\odot} / \mathrm{yr}$, mean far-IR luminosity, $\log \left(L_{\mathrm{FIR}}\right) \simeq 10.1$, and mean redshift 0.075 , together with a starburst population characterized by warm dust temperature (i.e., $\geq 36 \mathrm{~K}$ ), mean $\mathrm{SFR} \simeq 12 M_{\odot} / \mathrm{yr}$, mean far-IR luminosity, $\log \left(L_{\mathrm{FIR}}\right) \simeq 10.3$, and mean redshift 0.1 (Fig. 5).

\section{The $60 \mu \mathrm{m}$ luminosity function}

The observed flux $S_{v}$ is related to the rest-frame luminosity by:

$L_{v}=4 \pi D_{L}^{2} S_{v} / k(L, z)$,

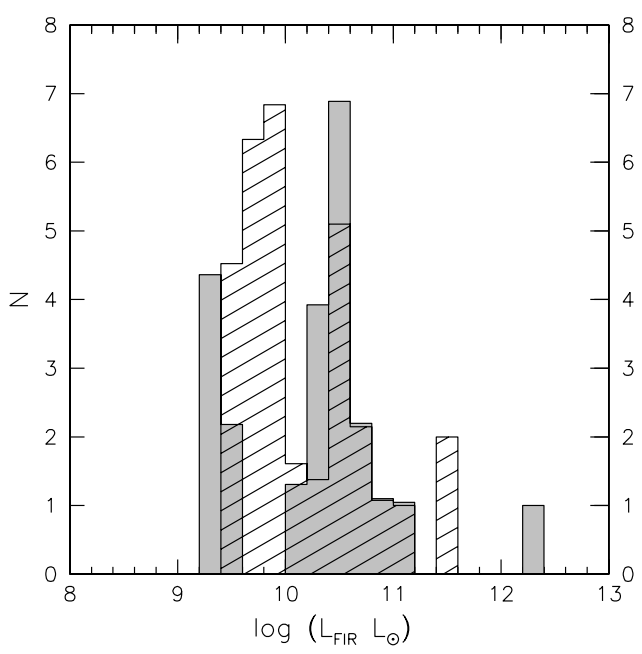

Fig. 3. The rest-frame far-infrared luminosity distribution for the IDS/ISOCAM sample of 56 galaxies. The separate distributions of warm (24 sources) and cold (32 sources) sub-populations are indicated with the (grey) open and (white) hatched histograms, respectively.
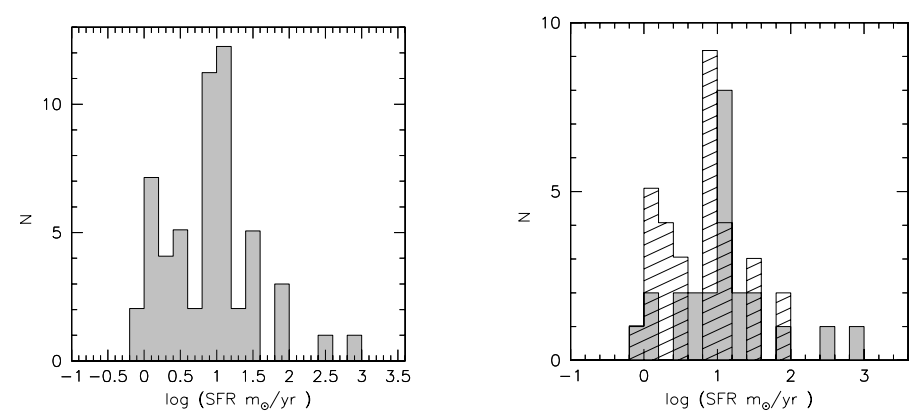

Fig. 4. Left: the SFR distribution for our sample of 56 sources. Right: the SFR distribution for warm, 24, and for cold, 32 sources, (grey) open and (white) hatched histograms respectively.

where $D_{L}$ is the luminosity distance, computed according to our cosmological model (Sect. 1), and $k(L, z)$ is the $K$-correction defined as:

$k(L, z)=\frac{(1+z) L_{v(1+z)}}{L_{v}}$

For the most distant galaxies $(z>0.2)$ the correction exceeds $20 \%$ of the luminosity. Figure 6 shows our derived $60 \mu \mathrm{m}$ differential luminosity function, i.e., the co-moving number density of sources per logarithmic luminosity interval, $\phi\left(L_{60}, z\right) \Delta$ $\log L_{60}$, where $L_{60}=v L_{v}$, calculated using the Schmidt-Eales $1 / V_{\max }$ estimator proposed by Schmidt (1968) and improved by Felten (1976) and Eales (1993). We assume Poisson errors, as tabulated by Gehrels (1986). $L_{60}$, i.e., the rest-frame luminosity, has been K-corrected as specified in Sect. 2.

According to the $V / V_{\max }$ test (Schmidt 1968), sources drawn from a population uniformly distributed in space should have a mean value of $V / V_{\max }$ equal to 0.5 . Larger (smaller) values of $V / V_{\max }$ are the result of a strong increase (decrease) of the co-moving space density of sources with redshift. We find $\left\langle V / V_{\max }\right\rangle=0.51 \pm 0.06$, consistent with a uniform distribution of sources. The IDS/ISOCAM sample is ten times deeper in flux density than the PSCz catalog and 100 times deeper than the IRAS $60 \mu \mathrm{m}$ Bright Galaxy Sample (Sanders et al. 2003), but no signatures of evolution arise from our sample. 


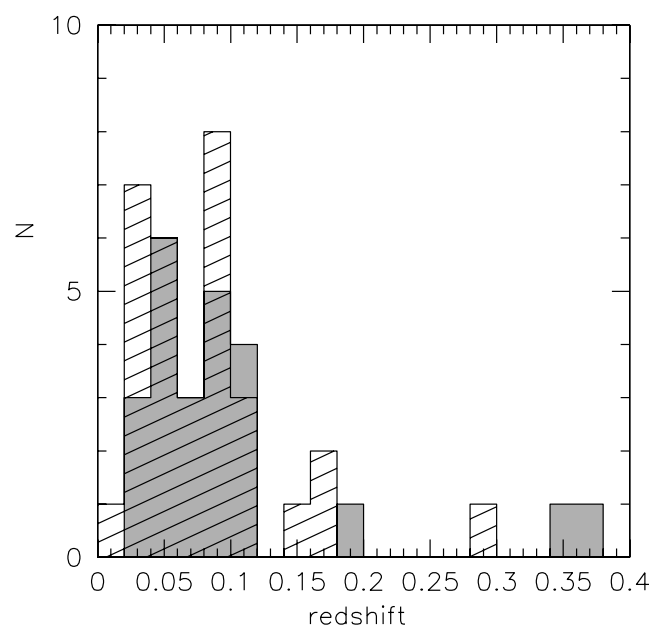

Fig. 5. The redshift distribution for warm, 24, and for cold, 32 sources, (grey) open and (white) hatched histograms, respectively.
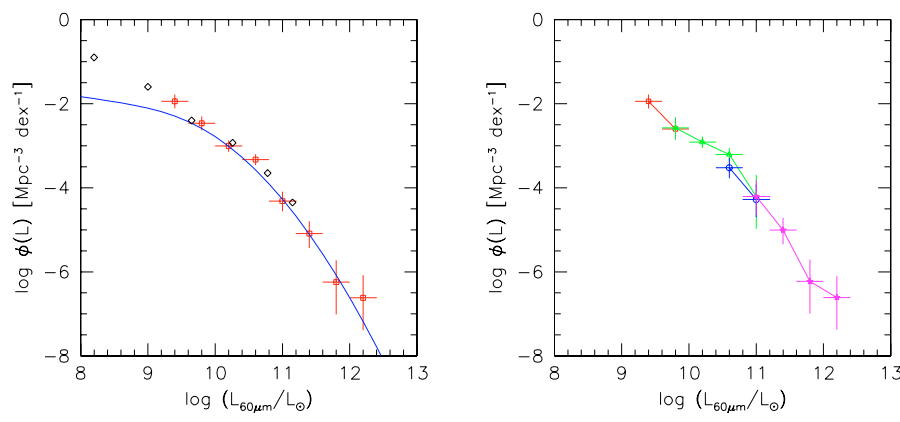

Fig. 6. Left: the rest-frame $60 \mu \mathrm{m}$ LF for our sample of 56 IDS/ISOCAM sources (red open squares) compared with LF of IRAS PSCz galaxies from Takeuchi et al. (2003, 2004; blue continuous line) and with LF of Spitzer extragalactic First Look Survey from Frayer et al. (2006) (black open diamonds). The bin size is $\Delta \log L_{60}=0.4$. Right: LF split into different redshift bins: asterisks (red) for $z<0.05$, triangles (green) for $0.05 \leq z<0.010$, open circles (blue) for $0.010 \leq$ $z<0.015$, and stars (magenta) for $z>0.15$.

The LF we derive is in good agreement with previous works based on the IRAS PSC (Saunders et al. 1990; Takeuchi et al. 2003, 2004). Takeuchi et al. (2003, 2004) revised the work by Saunders et al. (1990) by enlarging their galaxy sample to 15411 galaxies from the PSCz (Saunders et al. 2000) with a flux limit of $600 \mathrm{mJy}$ and a redshift range between 0 and 0.07 . Their analytic fit, shown in Fig. 6, is based on the same parameterization as Saunders et al. (1990):

$\phi\left(L_{v}\right)=\phi_{*}\left(\frac{L_{v}}{L_{*}}\right)^{1-\alpha} \exp \left[-\frac{1}{2 \sigma^{2}} \log _{10}^{2}\left(1+\frac{L_{v}}{L_{*}}\right)\right]$

with parameters: $\alpha=1.23 \pm 0.04, L_{*}=(4.34 \pm 0.86) \times$ $10^{8} h^{-2} L_{\odot}, \sigma=0.724 \pm 0.01$, and $\phi_{*}=(2.60 \pm 0.30) \times$ $10^{-2} h^{3} \mathrm{Mpc}^{-3}$ (Takeuchi et al. 2003, 2004).

Our results extend over three orders of magnitude in luminosity, from $\log \left(L_{60} / L_{\odot}\right) \simeq 9$ up to more than 12 . In the range where the samples overlap, our findings agree with the recent determination by Frayer et al. (2006, diamonds in Fig. 6), based on a complete sample of 58 sources with $S_{70}>50 \mathrm{mJy}$ and $z<0.3$, drawn from the Spitzer extragalactic First Look Survey. Despite the fact that the redshift range exceeds $z=0.3$, our LF does not show any evidence of evolution. A more direct test for evolution has been performed by computing LF in four different redshift
Table 1. The $60 \mu \mathrm{m}$ luminosity function: $V / V_{\max }$ in different redshift bins.

\begin{tabular}{rr}
\hline \hline Redshift bin & $V / V_{\max }$ \\
\hline $0.00 \leq z<0.05$ & $0.490 \pm 0.04$ \\
$0.05 \leq z<0.10$ & $0.471 \pm 0.08$ \\
$0.10 \leq z<0.15$ & $0.444 \pm 0.07$ \\
$z>0.15$ & $0.315 \pm 0.08$ \\
\hline
\end{tabular}

bins assuming no evolution (see Fig. 6, right panel, and Table 1). The results are fully consistent with this assumption.

\section{The $\mathbf{1 5} \mu \mathrm{m}$ luminosity function}

Deep surveys at $15 \mu \mathrm{m}$ carried out using ISO (i.e., Elbaz et al. 1999; Flores 1999; Lari et al. 2001; Metcalfe et al. 2003) seem to require strong evolution of $15 \mu \mathrm{m}$ sources starting from redshift 0.5 (see Lagache et al. 2005 and references therein). Several evolutionary models were developed to explain these results (e.g., Franceschini et al. 2001; Rowan-Robinson 2001), trying to fit IR/submillimeter source counts with different degrees of success. Nevertheless, none of them is based on a local LF obtained from $15 \mu \mathrm{m}$ data since the only available data until few years ago came from IRAS $12 \mu$ m photometry (Rush et al. 1993; Xu 1998; Fang et al. 1998).

A first attempt to build up the $15 \mu \mathrm{m}$ LF of a NEPR subsample was made by $\mathrm{Xu}$ (2000), using the bi-variate method to translate the $60 \mu \mathrm{m}$ local LF of IRAS galaxies (Saunders et al. 1990) to $15 \mu \mathrm{m}$. Its sample comprises 64 sources detected both at $60 \mu \mathrm{m}$ (Hacking \& Houck 1987) and at $15 \mu \mathrm{m}$ (Aussel et al. 2000), with redshifts measured by Ashby et al. (1996). Xu fitted the result obtained (see its Table 2 and Fig. 3), with a pure luminosity evolution model $L \propto(1+z)^{4.5}$. Nevertheless he said that it must be considered as a preliminary work, as discussed in Sect. 1.

A recent determination of the $15 \mu \mathrm{m}$ local LF was made by Pozzi et al. (2004) using the available data on the southern fields, S1 and S2, of the ELAIS survey (Oliver et al. 2000). Their data sample entails 150 galaxies with redshift $z \leq 0.4$, excluding sources classified as AGNs (both type one and type two). The $15 \mu \mathrm{m}$ LF was calculated with a parametric maximum likelihood method. Pozzi et al. (2004) separate spirals from starbursts using optical/mid-IR ratios, assuming that starbursts are the more mid-IR luminous galaxies with, on average, larger $\log \left(L_{15} / L_{R}\right)$ ratios. They estimate the $15 \mu \mathrm{m}$ local LF in the range $7.8 \leq \log \left(L / L_{\odot}\right) \leq 10.6$. Their findings are that the $15 \mu \mathrm{m} \mathrm{LF}$ of spirals is consistent with no evolution $\left(\left\langle V / V_{\max }\right\rangle=\right.$ $0.55 \pm 0.03)$, but that the value $\left\langle V / V_{\max }\right\rangle=0.64 \pm 0.03$ measured for the starbursts suggests that this population is in fact evolving.

\subsection{The bi-variate method}

Since our sample is flux limited at $60 \mu \mathrm{m}$, but not at $15 \mu \mathrm{m}$, we used the bi-variate method to calculate the $15 \mu \mathrm{m}$ LF of our sample. It was obtained by a convolution of the $60 \mu \mathrm{m} \mathrm{LF}$ with the $\log \left(L_{15} / L_{60}\right)$ distribution:

$\phi\left(\log L_{15}\right)=\int \phi\left(\log L_{60}\right) P\left(\log \left(L_{15} / L_{60}\right)\right) \mathrm{d}\left(\log L_{60}\right)$,

where $P\left(\log \left(L_{15} / L_{60}\right)\right)$ is the conditional probability function that gives the distribution of $\log \left(L_{15}\right)$ around the mean $15 \mu \mathrm{m}$ luminosity $\left\langle\log \left(L_{15}\right)\right\rangle$ at a given $60 \mu \mathrm{m}$ luminosity $\log \left(L_{60}\right)$. 
Table 2. Coefficients and parameters describing the distribution function, Pearson I curve of our sample.

\begin{tabular}{llllllllllll}
\hline \hline Case & $y_{0}$ & $a_{1}$ & $a_{2}$ & $m_{1}$ & $m_{2}$ & Mode & Mean & $\sigma$ & Skew & Kurt & $\chi_{v}^{2}$ \\
\hline All & 82.8 & 12.9 & 0.43 & 83.3 & 2.72 & -0.60 & -0.69 & 0.24 & 0.52 & 0.52 & 0.77 \\
$\sigma_{\mathrm{p}}$ & 16.5 & 1.56 & 0.04 & 10.2 & 0.39 & 0.04 & & & & & \\
\hline
\end{tabular}

Table 3. Parameters describing the LF of our sample.

\begin{tabular}{lllcc}
\hline \hline Case & $\phi_{*} / 10^{-3} \mathrm{Mpc}^{-3}$ & $L_{*} / 10^{8} L_{\odot}$ & $\alpha$ & $\sigma$ \\
\hline All & $5.32 \pm .40$ & $2.00 \pm .18$ & $1.241 \pm .021$ & $0.748 \pm .010$ \\
\hline
\end{tabular}

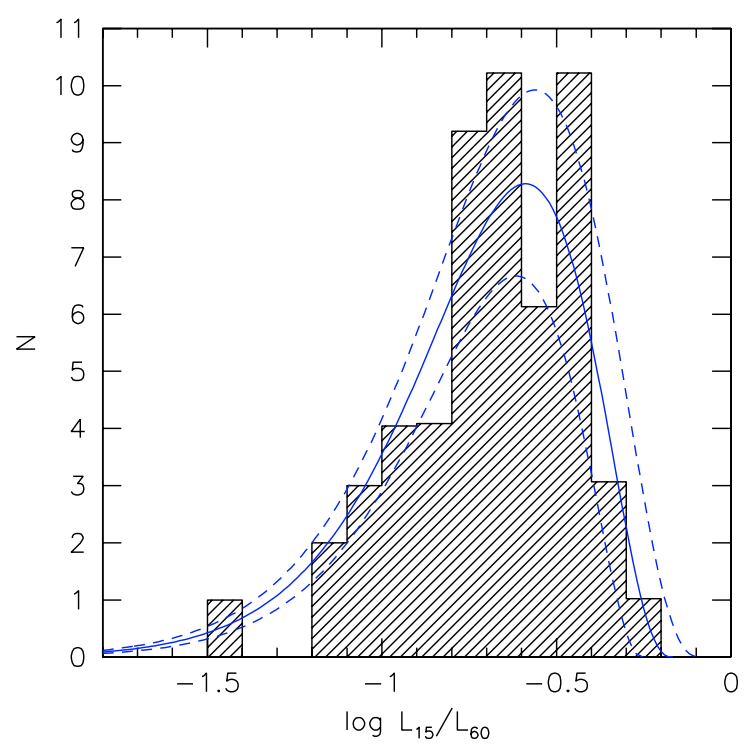

Fig. 7. Distribution of the $\log L_{15} / L_{60}$ ratio for 54 IDS/ISOCAM sources (see text). The bin size is $\Delta\left(\log L_{15} / L_{60}\right)=0.1$. The fitting function is a type I Pearson whose parameters, optimized following the LevenbergMarquardt method (see text), are reported in Table 2; dashed lines show the same curves with one $\sigma$ error in the parameters.

The distribution per unit interval of the logarithm of the luminosity ratio is well described by type I Pearson's curves (Pearson 1924; Elderton \& Johnson 1969):

$y=y_{0}\left(1+x / a_{1}\right)^{m 1}\left(1-x / a_{2}\right)^{m 2}$,

where $x=\log \left(L_{15} / L_{60}\right)$, with $-a_{1} \leq x \leq a_{2}$, and its origin is at the position of the peak of the distribution (mode). The values of the parameters are given in Table 2, together with the mean, the standard deviation $\sigma$, the skewness $\left(\mu_{3}^{2} / \mu_{2}^{3}\right)$, and the kurtosis $\left(\mu_{4} / \mu_{2}^{2}-3\right)$ of the distribution $\left(\mu_{i}\right.$ is the $i$ th moment about the mean). The quality of the fit is quantified by the value of $\chi^{2}$ per degree of freedom $\left(\chi_{v}\right)$, given in the last column and computed adopting the Levenberg-Marquardt method as implemented in Press et al. (1992); $\sigma_{\mathrm{p}}$ are the errors on the Pearson's parameters.

To perform these analyses we remove the sources 3-70 and 3-96 with AGN properties from our sample (see Sect. 2) and used the parametric solution of the $60 \mu \mathrm{m}$ LF derived by Takeuchi et al. $(2003,2004)$, which agrees well with our results (see Sect. 3, Eq. (3)).

Table 3 lists the parameters defining our $15 \mu \mathrm{m}$ local galaxy LF using the same parametric form as in Eq. (3) suggested by Pozzi et al. (2004). The Pozzi LF extends from 7.8 up to 10.6 in $\log \left(L_{15} / L_{\odot}\right)$, whereas our convolution extends over more than six orders of magnitude and 100 times deeper (Fig. 8). Our results agree with those by Pozzi et al. (2004) up to their

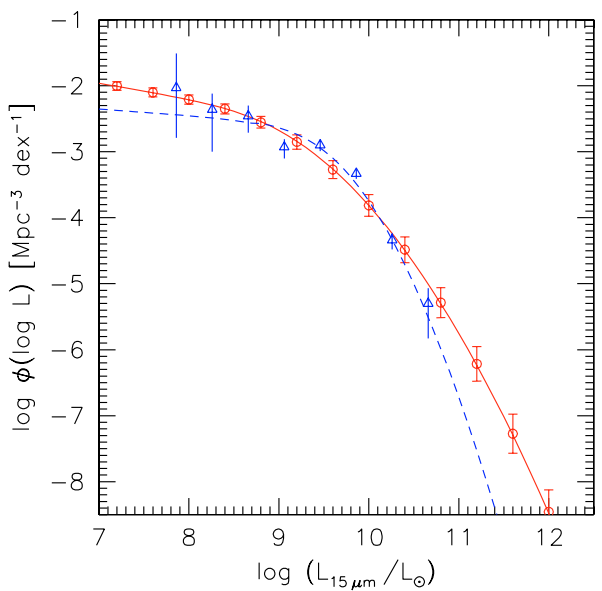

Fig. 8. The $15 \mu \mathrm{m}$ differential LF (red continuous line) for the IDS/ISOCAM sample (54 sources) compared with that of Pozzi et al. (2004) (blue dashed line and open triangles). The error bars correspond to one $\sigma$ error of the Pearson's curve parameters.

luminosity limit (10.6); however, beyond such a luminosity the two parametric LFs diverge so that at $\log \left(L_{15} / L_{\odot}\right)=11$ we expect 10 times more sources than Pozzi et al. (2004).

\subsection{Spiral and starburst populations}

Our sample includes 22 galaxies with rest-frame warm $L_{60} / L_{100}$ ratios and 32 galaxies with cold ratios, after excluding AGN (see Sect. 2). They define two different populations, starburst and spiral galaxies, respectively, as far as dust properties are concerned. Their distributions per unit interval of the logarithm of the luminosity ratios $\log \left(L_{15} / L_{60}\right)$, shown in Fig. 9 (left panel), are fitted well by Pearson's curves whose parameters are in Table 4. Their $15 \mu \mathrm{m}$ LF fits, i.e., Eq. (3), are in Fig. 9 (right panel), and the LF parameters are given in Table 5. We find that both populations contribute to the faint end of the LF. Spiral galaxies overcome starbursts by less than a factor of two. Such a factor is slightly reduced with luminosity, from 1.8 at $\log \left(L_{15} / L_{\odot}\right)=8$ to 1.3 at $\log \left(L_{15} / L_{\odot}=11.8\right)$. Our results differ from those of Pozzi et al. (2004), in particular predictions concerning starburst population. However, they used optical/midIR ratios instead of far-IR ratios to disentangle starbursts and spirals, assuming that starbursts are the more mid-IR luminous galaxies with, on average, $\operatorname{larger} \log \left(L_{15} / L_{R}\right)$ ratios.

To investigate this point further, we repeated our analysis using the same criterion as Pozzi et al. (2004) to discriminate starbursts from spirals. We used the APS catalog (http://aps .umn .edu) $R$-band magnitudes for the $37.5 \%$ of our sample galaxies that lacked them (see Sect. 1), applying a correction of -0.75 mag to bring the APS zeropoint into agreement with our own common sources. Figure 10 shows the restframe $L_{15} / L_{R}$ ratios vs. $L_{15}$ luminosity. From a least-squarefitting procedure we find:

$L_{15} / L_{R}=(0.64 \pm 0.04) \times L_{15}-(6.67 \pm 0.43)$ 
Table 4. Coefficients and parameters describing the distribution functions, Pearson I curves, of starburst and spiral populations.

\begin{tabular}{llllllllllll}
\hline \hline Case & $y_{0}$ & $a_{1}$ & $a_{2}$ & $m_{1}$ & $m_{2}$ & Mode & Mean & $\sigma$ & Skew & Kurt & $\chi_{v}^{2}$ \\
\hline Starbursts & 35.7 & 4.30 & 0.14 & 9.11 & 0.25 & -0.39 & -0.70 & 0.25 & 1.99 & 2.39 & 1.70 \\
$\sigma_{\mathrm{p}}$ & 12.3 & 1.55 & 0.02 & 3.56 & 0.18 & 0.002 & & & & & \\
Spirals & 43.2 & 1.47 & 0.32 & 4.43 & 0.19 & -0.64 & -0.64 & 0.23 & 0.05 & -0.84 & 0.77 \\
$\sigma_{\mathrm{p}}$ & 11.6 & 0.58 & 0.01 & 1.89 & 0.11 & 0.01 & & & & & \\
\hline
\end{tabular}

Table 5. Parameters describing the LFs of starburst and spiral populations.

\begin{tabular}{llccc}
\hline \hline Case & $\phi_{*} / 10^{-3} \mathrm{Mpc}^{-3}$ & $L_{*} / 10^{8} L_{\odot}$ & $\alpha$ & $\sigma$ \\
\hline Starbursts & $1.89 \pm .03$ & $2.96 \pm .03$ & $1.275 \pm .001$ & $0.740 \pm .015$ \\
Spirals & $3.36 \pm .07$ & $2.21 \pm .07$ & $1.236 \pm .006$ & $0.739 \pm .027$ \\
\hline
\end{tabular}
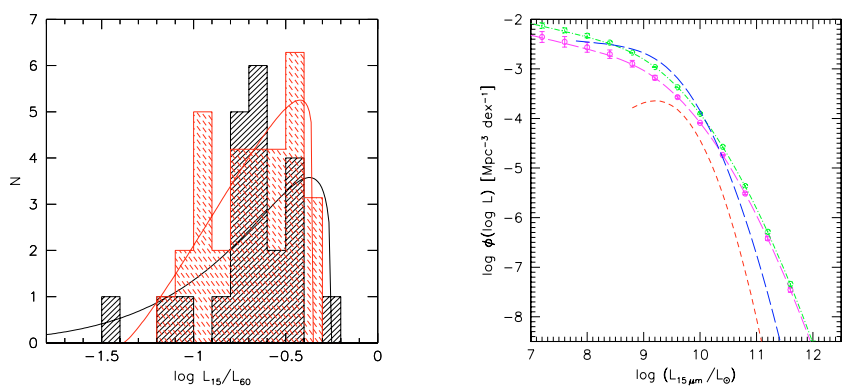

Fig. 9. Left: distribution of the $\log L_{15} / L_{60}$ ratios for starbursts (black continuous line), 22 sources, and spirals (red dashed line), 32 sources, selected on the basis of their far-IR colors. The bin size is $\Delta\left(\log L_{15} / L_{60}\right)=0.1$ and the parameters of the fitting functions, type I Pearson curves, are in Table 4 together with one $\sigma$ errors. Right: the contribution of such galaxy populations to the rest-frame $15 \mu \mathrm{m} \mathrm{LF}$ (see Table 5): starbursts (magenta long-dashed dotted line) and spirals (green dot-dashed line). Results are compared with those by Pozzi et al. (2004): starbursts (red short-dashed line) and spirals (blue long-dashed line).

with a dispersion of 0.20 dex. Our fit is steeper, 0.64 instead of 0.5, than that of Pozzi et al. (2004). Following Pozzi et al. (2004), we assume $L_{15} / L_{R}=-0.4$ to be the nominal separation between spiral and starburst populations. Warm and cold galaxies, selected on the basis of their far-IR colors, are mixed in Fig. 10. Such a criterion selects different galaxies in both the populations. Now there are 30 spirals and 24 starbursts in our sample. The distributions per unit interval of the logarithm of their luminosity ratios $\log \left(L_{15} / L_{60}\right)$, are fitted again by type I Pearson's curves (Fig. 11, left panel) whose parameters are in Table 6. Table 7 shows those of their $15 \mu \mathrm{m}$ LF fits (Fig. 11, right panel). Both such populations contribute to the faint end of the LF. The space densities of starbursts are just slightly greater than those of spiral galaxies.

\section{Conclusions}

Combining our observations (della Valle et al. 2006) with those by Ashby et al. (1996), we have reliable identifications and spectroscopic redshifts for $100 \%$ of the complete far-IR selected subsample comprising 56 IDS sources with $S_{60}>80 \mathrm{mJy}$ (Mazzei et al. 2001). The redshift distribution shows a tail extending up to $z \simeq 0.37$, in particular $\simeq 26 \%$ of the sources have redshifts $z>0.1$.

To fully exploit the potential of this sample, ten times deeper than the IRAS PSC, thus less liable to the effect of local density inhomogeneity, for investigating galaxy evolution, we

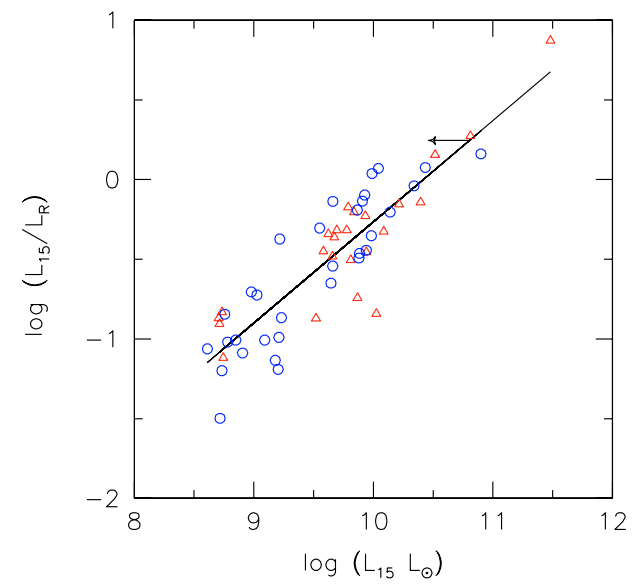

Fig. 10. The behavior of the $\log L_{15} / L_{R}$ ratio for warm sources (24, red triangles), and for cold sources (32, blue open circles) of IDS/ISOCAM sample; the continuous line corresponds to the leastsquare fit (see Eq. (6)).

calculate the $60 \mu \mathrm{m} \mathrm{LF}$ using the $1 / V_{\max }$ method. Current estimates are based on rather shallow samples. Even though our sample is five times deeper in redshift than the PSCz (Saunders et al. 2000) used by Takeuchi et al. (2003), our LF agree with their determination (Takeuchi et al. 2003, 2004) and with that by Frayer et al. (2006) based on a complete sample of galaxies with $S_{70}>50$ mJy drawn from the Spitzer extragalactic First Look Survey. Despite the fact that our redshift range exceeds $z=0.3$, and our $60 \mu \mathrm{m} \mathrm{LF}$ extends up to $\log L_{60}=12$, whereas that by Frayer et al. (2006) up to $\log L_{60} \simeq 11$, does not show any evidence of evolution. The $V / V_{\max }$ test gives a value consistent with a uniform distribution $\left(V / V_{\max }=0.51 \pm 0.06\right)$. Moreover, a more direct test for evolution has been performed by splitting the LF in different redshift bins assuming no evolution. The results are fully consistent with this assumption.

We present the bi-variate $15 \mu \mathrm{m} \mathrm{LF}$, one of the few determinations based on ISO data, by convolving the $60 \mu \mathrm{m}$ LF with the luminosity ratio distribution, $L_{15} / L_{60}$ of our sample. This extends to luminosity 100 times higher than before. Our result agrees with the recent determination by Pozzi et al. (2004) in the common range of luminosity, i.e., from 7.8 up to 10.6 in $\log \left(L_{15} / L_{\odot}\right)$. However, above $\log \left(L_{15} / L_{\odot}\right)=10.6$, the two parametric LFs diverge so that at $\log \left(L_{15} / L_{\odot}\right)=11$ we expect 10 times more sources than Pozzi et al. (2004). To investigate the role of galaxy populations on such a result, we disentangle starbursts and spirals on the basis both of their far-IR dust temperature, and of their $L_{15} / L_{R}$ ratios, as assumed by Pozzi et al. (2004). Such criteria select galaxies with different dust 
Table 6. Coefficients and parameters describing the distribution functions, Pearson I curves, of starburst and spiral populations.

\begin{tabular}{llllllllllll}
\hline \hline Case & $y_{0}$ & $a_{1}$ & $a_{2}$ & $m_{1}$ & $m_{2}$ & Mode & Mean & $\sigma$ & Skew & Kurt & $\chi_{v}^{2}$ \\
\hline Starbursts & 35.11 & 11.5 & 0.62 & 61.2 & 3.45 & -0.63 & -0.74 & 0.29 & 0.50 & 0.56 & 0.86 \\
$\sigma_{\mathrm{p}}$ & 10.7 & 2.29 & 0.12 & 12.3 & 0.74 & 0.11 & & & & & \\
Spirals & 60.3 & 10.0 & 0.33 & 47.8 & 0.94 & -0.60 & -0.67 & 0.21 & 0.41 & -0.34 & 1.03 \\
$\sigma_{\mathrm{p}}$ & 13.0 & 2.61 & 0.13 & 12.6 & 0.47 & 0.11 & & & & & \\
\hline
\end{tabular}

Table 7. Parameters describing the LF of starburst and spiral populations.

\begin{tabular}{lllcc}
\hline \hline Case & $\phi_{*} / 10^{-3} \mathrm{Mpc}^{-3}$ & $L_{*} / 10^{8} L_{\odot}$ & $\alpha$ & $\sigma$ \\
\hline Starbursts & $3.82 \pm .62$ & $1.81 \pm .45$ & $1.245 \pm .048$ & $0.761 \pm .028$ \\
Spirals & $2.92 \pm .88$ & $2.29 \pm .85$ & $1.234 \pm .084$ & $0.742 \pm .037$ \\
\hline
\end{tabular}
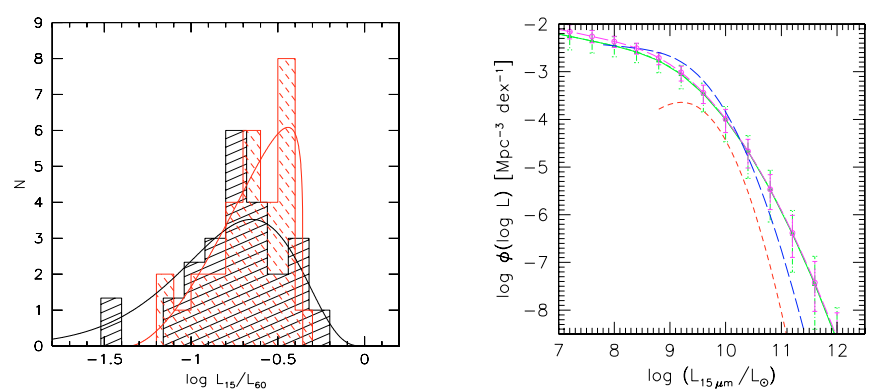

Fig. 11. Left: the distribution of the $\log L_{15} / L_{60}$ ratio for starbursts (black continuous line), 24 sources, and for spirals (red dashed line), 30 sources, selected with the same criterion as Pozzi et al. (2004). The bin sizes are $\Delta\left(\log L_{15} / L_{60}\right)=0.12$ and 0.1 , respectively. The parameters of the fitting functions, type I Pearson curves, are in Table 6. Right: the contribution of such galaxy populations to the rest-frame $15 \mu \mathrm{m} \mathrm{LF}$ (see Table 7): starbursts (magenta long-dashed dotted line) and spirals (green dot-dashed line). Our results are compared with those by Pozzi et al. (2004): starbursts (red short-dashed line) and spirals (blue longdashed line).

properties, as we point out in Sect. 4.2. Nevertheless, we find that both galaxy populations contribute to the faint end of the rest-frame $15 \mu \mathrm{m} \mathrm{LF}$, even though we adopt the same criterion as Pozzi et al. (2004). Moreover, in this case, above $\log \left(L_{15} / L_{\odot}\right)=$ 9 our findings are that starbursts and spirals give almost the same contribution to the mid-IR LF, whereas Pozzi et al. (2004) predict that spirals contribute 5-10 times more than starbursts.

Acknowledgements. We thank the referee, M. Ashby, for an unusually helpful and quick report, and Gianfranco de Zotti and Hervé Aussel for useful discussions. Our work was supported in part by MIUR (Ministero Italiano dell'Universitá e della Ricerca) and ASI (Ageanzia Spaziale Italiana).

\section{References}

Aussel, H., Coia, D., Mazzei, P., et al. 2000, A\&AS, 141, 257

Ashby, M., Hacking, P., Houck, J., et al. 1996, ApJ, 456, 428

Beichman, C. A., Neugebauer, G., Habing, H. J., et al. 1988, Infrared astronomi-

cal satellite (IRAS) catalogs and atlases. Volume 1: Explanatory supplement, NASA RP-1190

Bettoni, D., della Valle, A., Mazzei, P., et al. 2006, in prep.

Chapman, S. C, Scott, D., Syeidel, C. c, et al. 2000, MNRAS, 319, 318

della Valle, A., Mazzei, P., Bettoni, D., et al. 2006, A\&A, 454, 453

Désert, F.-X, Boulanger, F., \& Puget, J. L. 1990, A\&A, 237, 215

Devriendt, J. E. G., \& Guiderdoni, B. 2000, A\&A, 363, 851

Dole, H., Rieke, G. H., Lagache, G., et al. 2004, ApJS, 154, 93

Eales, S. 1993, ApJ, 404, 51

Elbaz, D., Cesarsky, C. J., Fadda, D., et al. 1999, A\&A, 351, L37
Elderton, W., \& Johnson, N. 1969, Systems of Frequency Curves (Cambridge University Press)

Fang, F., Shupe, D., Xu, C., \& Hacking, P. 1998, ApJ, 500, 693

Feigelson, E., \& Nelson, P. 1985, ApJ, 293, 192

Flores, H., Hammer, F., Thuan, T. X., et al. 1999, ApJ, 517, 148

Felten, J. E. 1976, ApJ, 207, 700

Franceschini, A., Mazzei, P., de Zotti, G., \& Danese, L. 1994, ApJ, 427, 140

Franceschini, A., Aussel, H., Cesarsky, C. J., et al. 2001, A\&A, 378, 1

Frayer, D. T., Fadda, D., Yan, L., et al. 2006, ApJ, 131, 250

Gehrels, N. 1986, ApJ, 303, 336

Guiderdoni, B., Hiven, E., Bouchet, F. R., \& Maffei, B. 1998, MNRAS, 295, 877 Hacking, P., \& Houck, I. R. 1987, ApJS, 63, 311

Hacking, P., Beichman, C. A., Condon, J. J., \& Houck, J. R. 1989, ApJ, 339, 12 Helou, G. 1986, ApJ, 311, L33

Helou, G., Khan, I., Malek, L., \& Boehmer, L. 1988, ApJS, 68, 151

Hopkins, A., Miller, J., Nichol, R., et al. 2003, ApJ, 599, 971

Isobe, T., \& Feigelson, E. 1990, Software Report: ASURV, The Pennsylvania State University, BAAS, 22, 917

Isobe, T., Feigelson, E., \& Nelson, P. 1986, ApJ, 306, 490

Kaplan, E., \& Meier, P. 1958, J. Am. Stat. Ass., 53, 457

Kennicutt, R. C. Jr. 1998, ARA\&A, 36, 189

Kewley, L., Gelle, M., Jansen, R., \& Dopita, M. 2002, ApJ, 124, 3135

Lagache, G., Puget, J.-L., \& Dole, H. 2005, ARA\&A, 43, 727

Lari, C., Pozzi, F., Gruppioni, C., et al. 2001, MNRAS, 325, 1173

Kauffmann, G., \& Charlot, S. 1998, MNRAS, 297, L23

Mazzei, P., de Zotti, G., \& Xu, C. 1992, A\&A, 256, 45

Mazzei, P., Curir, A., \& Bonoli, C. 1995, AJ, 422, 81

Mazzei, P., Aussel, H., Xu, C., et al. 2001, New Astr., 6, 265

Metcalfe, L., Kneib, J., McBreen, B., et al. 2003, A\&A, 407, 791

Neugebauer, G., Habing, H. J., \& van Duinen, R. 1984, ApJ, 278, L1

Oliver, S., Rowan-Robinson, M., Alexander, D., et al. 2000, MNRAS, 316, 749

Pearson, K. 1924, Tables for Statisticians and Biometricians (Cambridge University Press)

Pearson, C. P., Shibai, H., Matsumoto, T., et al. 2004, MNRAS, 347, 1113

Press, W. H., Teukolsky, S. A., Vetterling, W. T., \& Flannery, B. P. 1992, Numerical Recipes in FORTRAN: The Art of Science Computing, second edition (Cambridge University Press)

Pozzi, S., Gruppioni, C., Oliver, S., et al. 2004, ApJ, 609, 122

Rowan-Robinson, M. 2001, ApJ, 549, 745

Rowan-Robinson, M., \& Crawford, J. 1989, MNRAS, 238, 523

Rowan-Robinson, M., Helou, G., \& Walker, D. 1987, MNRAS, 227, 589

Rowan-Robinson, M., et al. 1999, in The Universe as Seen by ISO, ESA SP-427

Rush, B., Malkan, M., \& Spinoglio, L. 1993, ApJS, 89, 1

Sanders, D. B., \& Miralbel, I. F. 1996, ARA\&A, 34, 749

Sanders, D. B., Mazzarella, J. K., Kim, D., et al. 2003, AJ, 126, 1607

Saunders, W., Rowan-Robinson, M., Lawrence, A., et al. 1990, MNRAS, 242, 318

Saunders, W., Sutherland, W., Maddox, S., et al. 2000, MNRAS, 317, 55

Schmidt, M. 1968, ApJ, 151, 393

Soifer, B., Sanders, D., Madore, B., et al. 1987, ApJ, 320, 238

Takeuchi, T., Yoshikawa, K., \& Ishii, T. 2003, ApJ, 587, L89

Takeuchi, T., Yoshikawa, K., \& Ishii, T. 2004, ApJ, 606, L171

$\mathrm{Xu}$, C. 2000, ApJ, 541, 134

Xu, C., Hacking, P., Fang, F., et al. 1998, ApJ, 508, 576 